\title{
The Effect of Denervation and Treatment with the $\beta 2$-Agonist, Formoterol, on the Masseter Muscles of Growing Sprague Dawley Rats
}

\author{
Mayne $\mathbf{R J}^{1}$, Van der Poel $\mathbf{C}^{2}$, Woods $\mathbf{M G}^{3, *}$, and Lynch $\mathbf{G S}^{4}$ \\ ${ }^{1}$ Orthodontic Unit, University of Melbourne, Australia \\ ${ }^{2}$ Department of Physiology, Anatomy and Microbiology, Latrobe University, Australia \\ ${ }^{3}$ Department of Dentistry and Oral Health, Latrobe University, Australia \\ ${ }^{4}$ Department of Physiology, University of Melbourne, Australia
}

*Corresponding author: Woods MG, Department of Dentistry and Oral Health, Latrobe University, Australia, E-mail: m.woods@latrobe.edu.au

Received: 10 Jan, 2020 | Accepted: 16 Jan, 2020 | Published: 24 Jan, 2020

Citation: Mayne RJ, Van der Poel C, Woods MG, Lynch GS (2020) The Effect of Denervation and Treatment with the 32 -Agonist, Formoterol, on the Masseter Muscles of Growing Sprague Dawley Rats. Int J Dent Oral Health 6(1): dx.doi.org/10.16966/2378-7090.316

Copyright: (C) 2020 Mayne RJ, et al. This is an open-access article distributed under the terms of the Creative Commons Attribution License, which permits unrestricted use, distribution, and reproduction in any medium, provided the original author and source are credited.

\section{Abstract}

Aim: To determine the effects of denervation and treatment with the $\beta 2$-adrenoceptor agonist, formoterol, on the masseter muscle of the rat.

Hypothesis and rationale: Muscles in the craniofacial region, particularly the masseter muscle, are often subject to changes in size and strength as a consequence of post-surgical muscle wasting and neuromuscular disorders. These modifications can influence the timing and nature of orthodontic treatment, as well as post-orthodontic relapse.

Method: Young (4 week old) male Sprague Dawley rats were randomly assigned to one of four experimental groups: sham, denervated, denervated+ $\beta 2$-agonist, or $\beta 2$-agonist-only experimental groups. The rats in the denervated groups had their left masseteric nerve sectioned, and for those in the $\beta 2$ agonist groups, formoterol was injected directly into the left masseter muscle every 3 days, for 8 weeks. Muscle mass, muscle volume and muscle signal intensity (via MRI), muscle fibre Cross-sectional Area (CSA), and protein per mass of muscle were assessed.

Results: Surgical denervation of the masseter muscle resulted in a significant decrease in muscle mass, fibre CSA, muscle volume and an increase in MRI muscle signal intensity. These post-surgical changes were largely prevented by administration of formoterol, demonstrating that $\beta 2$-agonist administration can attenuate denervation-induced atrophy in the masseter muscle. Administration of a $\beta 2$-agonist alone without surgical denervation led to increases in muscle mass, muscle volume and fibre CSA, findings that are consistent with the well-reported anabolic effects of formoterol.

Conclusions: The findings indicate that intramuscular administration of a $\beta 2$-agonist can attenuate muscle atrophy concomitant with denervation. Such an approach may have clinical application in orthodontics for helping regulate muscle architecture and function in the treatment of orofacial disorders.

\section{Introduction}

The muscles of mastication, particularly the masseter muscle, play a major role in speech, digestion and respiration, and provide the principal driving forces for the movement of the mandible. These muscles are also acknowledged to play a major role in overall facial growth and in orthodontic and orthognathic surgery treatment $[1,2]$. Health and normal function of these muscles of mastication have a direct influence on quality of life. Patients who suffer craniofacial problems such as hemifacial microsomia [3], muscular dystrophy [4,5], or other craniofacial deformities, usually require surgical correction. There is a general assumption that the muscles of mastication have similar properties, but in fact they differ considerably. The location, shape and size of the muscles of mastication vary between patients and so providing new knowledge about these muscles is useful for the planning of orthodontics and maxillofacial surgery [4,6-8]. The malleability of the growing craniofacial muscles to changes in size and strength is largely unknown. The use of pharmacological agents that exert anabolic effects on skeletal muscle may have therapeutic application for neuromuscular disorders to increase muscle strength and size of the oro-facial muscles. Age-related muscle wasting can affect the muscles of mastication and influence the consistency of food intake, and indirectly affect health and quality of life. Atrophy of the orofacial muscles, particularly the masseter muscle, has been associated with ageing and edentulism [5]. In clinical orthodontics, an intervention that could control muscle size and strength during treatment might help promote favourable outcomes in the years following orthodontic treatment.

\section{$\beta 2$-agonists}

$\beta 2$-adrenoceptor agonists ( $\beta 2$-agonists) were originally developed for use in asthma patients to promote bronchodilation. However, it became apparent that $\beta 2$-agonists can also have muscle growthpromoting effects $[9,10]$, and a "repartitioning" [11] where the increases in muscle mass are accompanied by a concomitant decrease in body fat [12]. Not surprisingly, these effects of $\beta 2$-agonists were 
of interest to the livestock industry in their attempts to improve meat quality and feed efficiency. They were also quickly used or abused by athletes to gain a competitive advantage in strength and power sports, and subsequently banned by the International Olympic Committee (IOC). Due to their specific muscle anabolic actions, $\beta 2$-agonists may also have potential therapeutic applications for muscle wasting conditions.

\section{Uses of $\beta 2$-agonists}

Animal models have been used extensively to examine the potential for $\beta 2$-agonists to combat the muscle wasting associated with ageing (sarcopaenia) [13,14], cancer cachexia [15], sepsis, inactivity, unloading or microgravity [16], chronic obstructive pulmonary disease [17], muscular dystrophies [18,19], and other neuromuscular disorders [20]. An excellent experimental model for investigating muscle atrophy is denervation, which involves removal of a section of the nerve supplying a muscle and subsequent loss of muscle mass $[18,21,22]$. In conditions such as sarcopenial [4] and the muscular dystrophies [19], where muscle mass and strength are decreased, systemic administration of $\beta 2$-agonists has been shown to have therapeutic potential [11,23-25]. Clinical trials involving the systemic administration of $\beta 2$-agonists for neuromuscular disorders have reported improved muscle strength in some patients [26,27]. However, systemic administration of $\beta 2$-agonists can have deleterious cardiovascular side effects [28], especially after chronic long term treatment [29,30], resulting in cardiac hypertrophy [31], heart congestion and interstitial oedema, and impaired cardiac function characterized by a decrease in cardiac output, tachycardia, palpitations and ischemia $[30,32]$. Other side-effects reported include insomnia, cramps, tremors, and nervousness [26]. Most of these effects are dose specific, but they have so far limited the clinical use of $\beta 2$ agonists for muscle wasting diseases since the doses required to cause skeletal muscle hypertrophy have exceeded the estimated safe limits in humans [33]. A more recently synthesized $\beta 2$-agonist, formoterol, has the advantage of having an increased duration of action as well as increased $\beta 2$-adrenoceptor selectivity [34] compared with traditional older generation $\beta 2$-agonists such as fenoterol and clenbuterol [35]. At equimolar doses, formoterol has been shown to be more efficacious than clenbuterol for increasing skeletal muscle mass and force producing capacity [32]. Formoterol administration to rats (1.0 and $2.0 \mathrm{mg} / \mathrm{kg} /$ day) results in significant skeletal muscle hypertrophy, with reduced effects on the heart [36].

\section{Investigation of muscle properties}

The development of non-invasive imaging techniques such as CT (Computed Tomography) scanning, MRI (Magnetic Resonance Imaging) [4,37] and ultrasonography, enable fast and accurate imaging of muscle size and volume changes in vivo. Signal Intensity (SI) is a measure of tissue contrast differences. MRI is the ideal tool for imaging skeletal muscle and can identify characteristic signal intensity increases corresponding to changes following muscle denervation [3840] skeletal muscle demonstrates characteristic MRI signal intensity patterns in different stages of muscle denervation. MRI investigation of denervated muscles was first described by Polak JF, et al. [38].

To date, the effects of intramuscular administration of $\beta 2$-agonists with or without muscle atrophy have not been reported for craniofacial muscles. Intramuscular injection of $\beta 2$-agonists allows for site-specific drug delivery that can minimize or obviate deleterious effects on the heart usually associated with systemic administration.

The aim of this study was to examine whether denervation atrophy of the masseter muscle could be attenuated by low dose intramuscular administration of the $\beta 2$-agonist, formoterol.

\section{Methods and Materials}

All experiments were approved by the Animal Experimentation Ethics Committee of The University of Melbourne (AECC number 0704146.1), and Howard Florey Institute Animal Experimentation Ethics Committee (AEC number 07-067), and performed in accordance with the guidelines determined by the National Health and Medical Research Council.

\section{Animals}

Four week old male Sprague Dawley rats (70-140g) were housed in standard cages in the Biological Research Facility at The University of Melbourne. The animals were kept under a 12:12 hour light dark cycle (light 0600-1800) with free access to food (rat chow) and water ad libitum. All rats were randomly assigned to either a surgical sham $(n=5)$, denervated only group $(n=9)$, denervated $+\beta 2$-agonist $(n=8)$, or $\beta 2$-agonist-only $(n=9)$ groups.

\section{Experimental procedure}

Rats were anaesthetised with an intraperitoneal (i.p) injection of a mixture of Ketamine $(225 \mathrm{mg} / \mathrm{kg})$ and Xylazine $(30 \mathrm{mg} / \mathrm{kg})$, with supplemental doses administered as needed to maintain an appropriate depth of anaesthesia, such that animals did not respond to tail or toe pinch.

In the surgical sham and denervated groups, a small (3-10mm) incision was made over the area below and directly parallel to the zygomatic arch. The left side of the rat was arbitrarily chosen as the "experimental" side in all animals. The platysma and masseter muscle fibres were gently parted and the masseteric nerve identified near the sigmoid notch of the coronoid process of the mandible (Figure 1). In the denervated groups, the masseter muscles were surgically denervated through cutting and removal of a $5 \mathrm{~mm}$ section of the masseteric nerve and its branches en masse. In the surgical sham group, the masseteric nerve was identified but not cut. In the denervated $+\beta 2$ agonist group, the masseter muscle was injected i.m with formoterol $(100 \mu \mathrm{g}$ in saline; Astra-Zeneca, Molndal, Sweden) at the time of surgery. The incision was closed with silk suture. Surgery was not performed on the $\beta 2$-agonist-only group. Every 3 days, the $\beta 2$-agonist-only and denervated $+\beta 2$-agonist groups were administered an i.m injection of formoterol $(100 \mu \mathrm{g}$ in saline) for a period of 8 weeks. The first 4-12 weeks of life is a period of rapid growth of the craniofacial area in growing rats [41].

To perform the i.m injection, the rats were anaesthetized by placing them in a clear plastic drop box, ventilated with $5 \%$ Isoflurane $((1 \mathrm{ml} /$ $\mathrm{ml})$ CENVET, Australia) in a 1:1 mix of medical-grade air and oxygen. Once anaesthetized, a nose cone was placed over the rat's snout to maintain anesthesia using an inhaled gas machine (Mediquip, Australia) which supplied 2-2.5\% Isoflurane (0.5L/min). Animals were anaesthetized for approximately 2-3 minutes, sufficient for the i.m injection of formoterol to be administered.

\section{Evaluation of muscle changes}

At the end of the experimental period ( 8 weeks), the animals were anaesthetized and placed in an MRI machine to measure changes in muscle volume and signal intensity. The animal MRI machine consisted of a Bruker Biospec 4.7 Tesla BIOSPEC system MR 47/30 DBX MRI/MRS (Bruker, Germany). For the purpose of keeping the rat motionless while in the MRI scanner, anesthesia was induced by placing the rat in a clear plastic box, ventilated via a Colombus CIV101 ventilator, with $5 \%$ Isoflurane in a 1:1 mix of compressed medical grade air and oxygen. Once the rat was anaesthetized, it was placed 
A

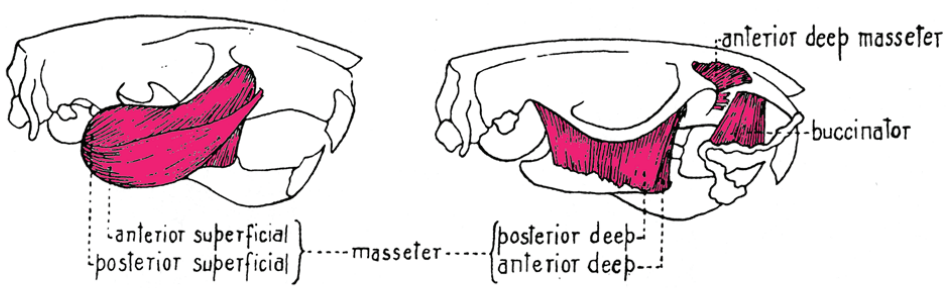

C

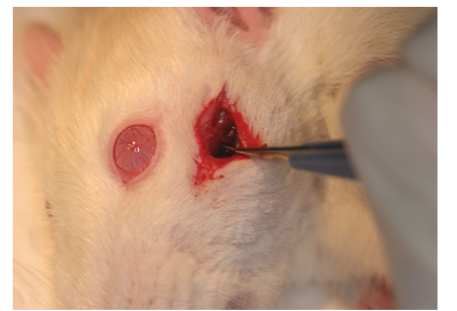

D

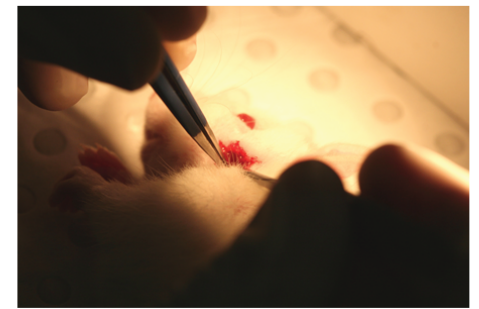

B

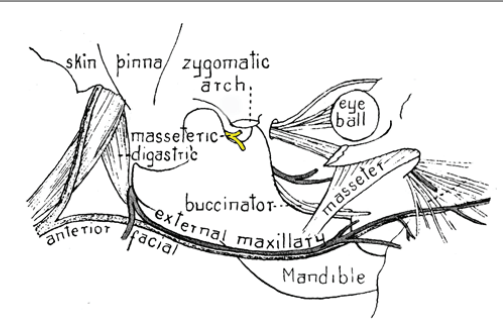

E

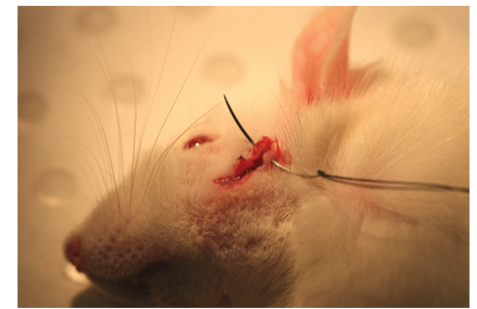

Figure 1: Example anatomical diagrams of rat craniofacial region.

A: The relative size of the masseter muscle on the rat skull showing the four main portions, the anterior and posterior superficial; and anterior and posterior deep, B: The masseteric nerve (in yellow) as it passes over the sigmoid notch of the mandible of the rat, C and D: example of 3-10mm of the incision made to access the nerve as described, E: the incision was closed with a black silk suture as required. (Pictures A and B were adapted from Green, EC, Anatomy of the rat, Hafner publishing, 1968) [12].

in an MR-compatible head holder with a nose cone placed over the snout to maintain anesthesia with $2-2.5 \%$ Isoflurane. The respiratory rate was monitored continuously throughout the experiment using PowerLab 8-channel physiological monitoring and recording systems (AD Instruments, Australia) with a probe placed under the surface, ventral to the monitoring system interfaced on a Silicon Graphics Octane computer (Silicon Graphics Co. Inc., Mountain View, CA, USA.) using acquisition and monitoring software Paravision (Bruker, Germany). Axial and coronal T2-weighted images were acquired. Axial T2-weighted images were acquired using a RARE (fast spin echo) sequence with repetition time (TR) $6212.8 \mathrm{~ms}$, echo time (TE) $62.7 \mathrm{~ms}$, field of view (FOV) $50 \times 50 \mathrm{~mm}$ with $256 \times 256$ matrix, 44 slices and slice thickness of $1 \mathrm{~mm}$, scanning time equal to $13 \mathrm{~m} 15 \mathrm{~s}$ for 4 NEX (NEX is the square root of the number of acquisitions). Coronal T2-weighted images were acquired using a RARE sequence with TR $6200 \mathrm{~ms}$, TE $62.7 \mathrm{~ms}$, FOV $55 \times 55 \mathrm{~mm}$ with $256 \times 256$ matrix, 32 slices and slice thickness of $1 \mathrm{~mm}$, scanning time equal to $13 \mathrm{~m} \mathrm{13s}$ for 4 NEX (Figure 2).

Data from the MRI was saved in DICOM format (DCM) for later 3D reconstruction and analysis of volume using software package AMIRA 4.1 (TGS Template Graphics Software, Inc., USA). Using AMIRA, the boundaries of the masseter were manually outlined in each slice. The interval between outlined areas was $1 \mathrm{~mm}$. The software reconstructed the complete muscle image comprised of the outlined areas multiplied by the slice, giving an extrapolated volume of the muscle. Mean signal intensity was calculated for the same region adjacent to the condylar cartilage (Figure 3 ) in both masseter muscles for each rat and displayed as an arbitrary unit. The measurements were taken on two different occasions one week apart, and calculated as an average. The ratio of signal intensity and volume was calculated for each muscle. SI is a function of the proton density, the decay time of the magnetization along the axis of the main magnetic field (T1), the decay time of the magnetization in the transverse plane (T2) and the MR machine parameters repetition time (TR) and echo time (TE). Signal intensity measurement with the spin-echo method is determined according to the following formula: $\mathrm{I}=\mathrm{N}(\mathrm{H}) \times \mathrm{e}(-\mathrm{TE} / \mathrm{T} 2) \times(1-\mathrm{e}[-\mathrm{TR} / \mathrm{T} 1])$, where $\mathrm{I}$ is signal intensity, $\mathrm{N}(\mathrm{H})$ is proton density, TE is echo time (msec), and $\mathrm{TR}$ is repetition time (msec). If parameters TR and TE are unchanged, decreased $\mathrm{T} 1$ values, increased $\mathrm{T} 2$ values, and higher proton density values increase the signal intensity [39]. Acutely denervated muscles show a high signal intensity pattern on T2-weighted sequences and normal signal intensity on T1-weighted MRI images [39]. Muscle atrophy demonstrates high signal changes on T1-weighted sequences in association with volume loss [40]. MR imaging can also depict muscle conditions such as muscular dystrophy, muscle injury, and age-related muscle changes. After the MRI acquisition, with the rat still anaesthetised, the animal was killed by a lethal dose of Nembutal $(100 \mathrm{mg} / \mathrm{kg})$ administered i.p.

Both left and right masseter muscles and the heart were immediately excised and trimmed of tendons and adherent connective tissue, blotted once on filter paper, and then weighed on an analytical balance. Immediately after weighing, each muscle was snap-frozen in thawing isopentane, in liquid nitrogen and stored at $-80^{\circ} \mathrm{C}$ for later histological and biochemical examinations.

\section{Error of the method}

Dahlberg error [42] was calculated for volume measurement. To determine error in the method of volume measurement from the MRI, duplicates of the volume measurements of a random sample rat left and right masseter muscles $(n=5)$ were taken at two different time-points, one week apart. Dahlberg error [42] was calculated for all volume measurements, and paired t-tests used to compare measurements at the two time intervals. The Dahlberg error was small with an average error of $0.141 \mathrm{~cm}^{3}$. The range of error was $0.0-0.22 \mathrm{~cm}^{3}$ for volume measurements. No differences ( $\mathrm{p}>0.05)$ between the measurements were calculated with a paired t-test, and so all measurements were included in the study.

\section{Histological examination}

Each frozen muscle sample was sectioned transversely as close to the mid-belly region of the masseter muscle as possible using a cryostat 

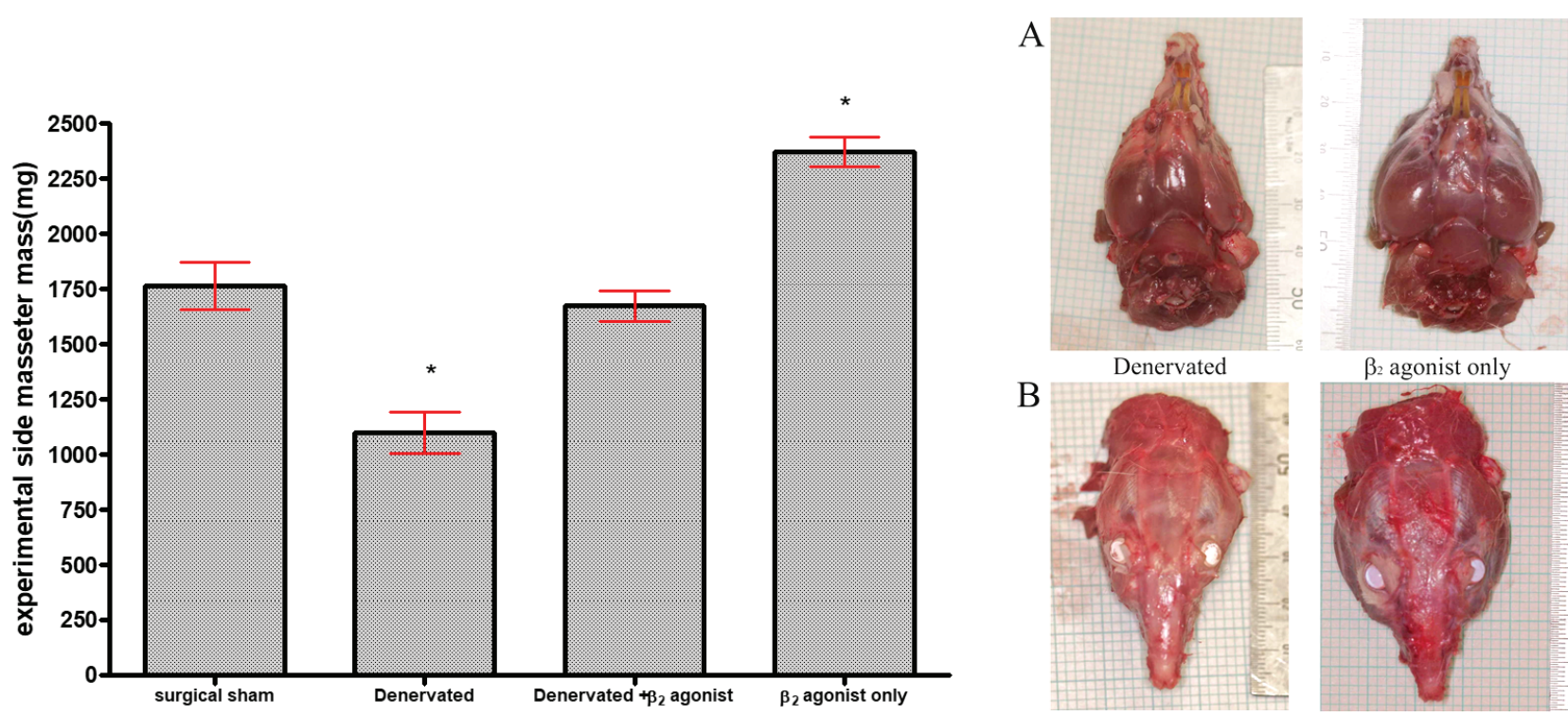

B

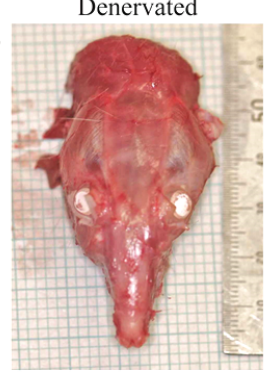

$\beta_{2}$ agonist only

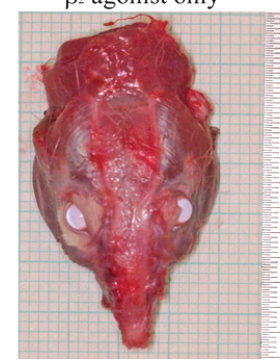

Figure 2: Graph of experimental (LHS) masseter muscle mass (mg).

Mass significantly decreased in the denervated group, significantly increased in the $\beta 2$ agonist group, and not changed in the denervated $+\beta 2$ agonist group. ${ }^{*} p<0.05$ in experimental compared to surgical sham (control). A: On left picture the denervated masseter is noticeably smaller than the control side, and noticeably smaller than the $\beta 2$ agonist group animal on the right. B: The same animals from above.

microtome at $-20^{\circ} \mathrm{C}$. Serial sections $(8 \mu \mathrm{m}$ thick) were placed onto an uncoated glass microscope slide and stained with Haematoxylin and Eosin (H\&E) to determine general muscle architecture and muscle fibre Cross-sectional Area (CSA). Images of the muscle were acquired with a digital camera (Spot model 1.3.0, Diagnostic Instruments, Sterling Heights, MI, USA) attached to an upright microscope (Olympus B $\times$ 51 light microscope, Olympus, Tokyo, Japan) at $\times 10$ magnification. The images were then analysed in a single blinded manner, using a calibrated Analytical Imaging Station (AIS, v6.0, imaging research, Ontario, Canada). The mean CSA of individual muscle fibres was calculated by interactive determination of the circumference of 150 adjacent fibres from the centre of every muscle section.

\section{Muscle protein analysis}

To determine the protein content in the masseter muscle, a sample of muscle was homogenized in a suitable buffer and total protein per mass of muscle was determined using a DC assay (Biorad, Virginia, USA), with bovine serum albumin standards. Protein assays were completed in triplicate in 96-well microplates (Nalge Nunc International, New York) and read on a multiscan spectrum microplate spectrometer, running Multiskan Spectrum Software (V1.0, Thermo Electron Corporation, Milford, MA, USA).

\section{Statistical analysis}

All values are expressed as mean \pm Standard Error of the Mean (SEM) unless otherwise specified. Experimental groups were compared (SPSS v16 for Windows, SPSS Inc. Chicago, Illinois, USA) using a one-way analysis of variance ANOVA (Tukey HSD) for the effect of surgery, formoterol and denervation, or paired t-tests to compare left and right sides to each other. Differences between groups were considered significant when $\mathrm{p}<0.05$. CSA data were analysed for normality using the Anderson-Darling Normality Test. Where muscle fibre CSA was not normally distributed, the $95 \%$ confidence interval of the median was used. Differences were considered significant when no overlap existed between the $95 \%$ confidence interval of the median. Pearson's (2-tailed) correlations were used to determine the relationship between mass measurements, volume measurements, and signal intensity.

\section{Results \\ Effect of surgical procedure}

The surgical procedure (sham surgery group) and i.m injections every three days did not affect the feeding, growth, behavior or health of the rats as evidenced from the similar body and heart masses at the end of the experimental period. Sham surgery did not affect masseter muscle mass on experimental and control sides, consistent with previous studies [43].

\section{Body mass}

Animals were initially age matched and randomly assigned to treatment groups. Prior to commencing the experiment, there was a significant difference $(p<0.05)$ between the initial body weights of the surgical sham compared with the $\beta 2$-agonist-only group; however, no differences existed between any other groups. At the end of the experiment there was no difference in body mass. Body mass of the surgical sham, denervated, and denervated $+\beta 2$-agonist, and $\beta 2$ agonist only groups all increased over the 8 week experimental period. The average increase in body mass over the experimental period was $480 \%$. There were no differences in the increase in mass between the groups with all groups growing at similar rates over the 2 month period (Table 1).

\section{Heart mass}

There was no difference in heart mass between groups over the 8 week experimental period ( $>0.05$ ) (Table 1 ). A major side effect of systemic $\beta 2$-agonist administration is an increase in heart mass resulting in severe heart defects. In this study utilising intramuscular 
Table 1: Summary of data for Sprague Dawley rats after 2 month experimental period.

\begin{tabular}{|c|c|c|c|c|c|c|c|c|}
\hline & \multicolumn{2}{|c|}{ Surgical sham (control) } & \multicolumn{2}{|c|}{ Denervated } & \multicolumn{2}{|c|}{ Denervated+ $\beta 2$ agonist } & \multicolumn{2}{|c|}{$\beta 2$-agonist } \\
\hline & Mean & SEM & Mean & SEM & Mean & SEM & Mean & SEM \\
\hline IM (mg) & 11560 & \pm 1263.56 & 9800 & \pm 436.84 & 9700 & \pm 252.21 & 9262.5 & \pm 312.78 \\
\hline EM (mg) & 50574.1 & \pm 2098.68 & 46860 & \pm 1126.44 & 47090 & \pm 1315.39 & 47123.75 & \pm 1236.33 \\
\hline BMI (\%) & 459.50 & \pm 54.53 & 482.95 & \pm 15.41 & 483.97 & \pm 22.64 & 512.57 & \pm 11.68 \\
\hline $\mathrm{HM}$ (mg) & 1420.52 & \pm 80.35 & 1322.67 & \pm 75.90 & 1382.35 & \pm 40.13 & 1377.38 & \pm 33.53 \\
\hline HM/EM (\%) & 2.80 & \pm 0.07 & 2.82 & \pm 0.13 & 2.99 & \pm 0.07 & 2.88 & \pm 0.06 \\
\hline LMM (mg) & 1763.50 & \pm 107.73 & 1097.42 & $\pm 95.52 * \dagger$ & 1673.01 & $\pm 70.53 \dagger$ & 2368.77 & $\pm 67.02 *$ \\
\hline RMM (mg) & 2022.98 & \pm 53.22 & 1776.56 & \pm 63.39 & 2209.61 & \pm 70.74 & 2311.48 & \pm 56.78 \\
\hline $\operatorname{LV}\left(\mathrm{cm}^{3}\right)$ & 1.405 & \pm 0.076 & 0.897 & $\pm 0.066^{*}+$ & 1.513 & $\pm 0.067 \dagger$ & 2.115 & \pm 0.084 \\
\hline $\mathrm{RV}\left(\mathrm{cm}^{3}\right)$ & 1.63 & \pm 0.056 & 1.423 & \pm 0.057 & 1.988 & \pm 0.078 & 2.038 & \pm 0.097 \\
\hline SIL & 23.156 & \pm 0.623 & 27.201 & $\pm 1.279^{*}$ & 26.518 & \pm 0.727 & 22.588 & \pm 0.502 \\
\hline SIR & 21.599 & \pm 0.538 & 23.512 & $\pm 0.793+$ & 22.99 & \pm 0.535 & 22.4 & \pm 0.554 \\
\hline $\operatorname{CSAL}\left(\mu \mathrm{m}^{2}\right)$ & 378.23 & \pm 8.464 & 217.38 & $\pm 5.434^{*}$ & 374.79 & \pm 8.708 & 513.81 & $\pm 8.095^{*}$ \\
\hline PCL (mg/g) & 0.061 & \pm 0.005 & 0.057 & \pm 0.004 & 0.056 & \pm 0.005 & 0.060 & \pm 0.006 \\
\hline
\end{tabular}

IM: Initial Body Mass; EM: End Body Mass; BMI: Percentage Increase in Body Mass; HM: Heart Mass; HM/EM: Ratio Heart weight/end body weight; LMM: Experimental Side (LHS) Masseter Mass; RMM: Control Side (RHS) Masseter Mass; LV: Experimental Side Masseter Volume; RV: Control Side (RHS) Masseter Volume; SIL: Mean Signal Intensity from Experimental Side (LHS) MRI; SIR: Mean Signal Intensity from Experimental Side (RHS) MRI; CSAL: Median Cross Sectional Area of Experimental Side (LHS) Masseter; PCL: Total Protein Concentration from Experimental Side (LHS) Masseter Muscle. Results presented as Mean \pm SEM

*p $<0.05$ significant difference compared to same surgical sham (control) (LHS)

$+p<0.05$ significant difference compared to control side (RHS)

administration, when heart mass was normalised to body mass (Table 1), there was no difference between any groups over the 8 week period.

\section{Masseter muscle mass}

The muscle mass of the denervated group was significantly different to all other groups $(\mathrm{p}<0.05)$. Denervation resulted in a $38 \%$ decrease in masseter muscle mass compared with surgical sham controls (Table 1). Denervated $+\beta 2$-agonist group masseter muscle mass was maintained at surgical sham controls (Figure 2). Administration of $\beta 2$-agonistonly resulted in a $36 \%$ increase in masseter muscle mass, which was significantly different from all other groups $(\mathrm{p}<0.05)$.

Muscle mass of the denervation group and denervation $+\beta 2$-agonist group was significantly decreased compared with the contralateral side $(\mathrm{p}<0.05)$. Denervation, and denervation $+\beta 2$-agonist groups had decreases in muscle mass of $38 \%$ and $24 \%$, respectively compared with the contralateral controls. Muscle mass of $\beta 2$-agonist-only and Sham surgery groups were not different from their contralateral control $(\mathrm{p}>0.05)$.

\section{Masseter muscle volume}

Denervation resulted in a significant $56 \%$ decrease $(\mathrm{p}<0.05)$ in masseter muscle volume compared with the surgical sham as measured on MRI (Table 1, Figure 3). Denervation $+\beta 2$-agonist masseter muscle volume was not different from controls. $\beta 2$-agonist only group masseter muscle volume was increased by $50 \%$ compared with the surgical sham controls $(\mathrm{p}<0.05)$.

When comparing left and right side masseter muscle volumes (Table 1 ), the sham surgery, denervation $+\beta 2$-agonist denervation left side volumes were significantly decreased compared with contralateral volumes $(p<0.05)$. $\quad \beta 2$-agonist only group left masseter volume was significantly larger than the right side masseter muscle volume $(\mathrm{p}<0.05)$.

\section{Signal intensity}

Masseter muscles of the denervated group had a $15 \%$ increase in mean SI $(\mathrm{p}<0.05)$ on a T2-weighted MRI, compared with control (Table 1, Figure 4 ). The denervated $+\beta 2$-agonist group SI was maintained at surgical sham group (control) values. The $\beta 2$-agonistonly group was not different from control. When comparing left to right side signal intensity changes in the masseter muscle, the sham surgery, denervation and denervation $+\beta 2$-agonist all resulted in significant changes in signal intensity $(\mathrm{p}<0.05)$. The $\beta 2$-agonist only group showed no difference in signal intensity between sides.

\section{Muscle CSA}

Muscle fibre Cross-sectional Area (CSA) varied significantly between groups (Table 1, Figure 5). Denervation resulted in a significant decrease in median muscle CSA compared with the surgical sham $(\mathrm{p}<0.05)$. Denervation $+\beta 2$-agonist group was maintained at control values ( $p>0.05)$. $\beta 2$-agonist administration resulted in a $29 \%$ increase in CSA compared with the surgical sham $(\mathrm{p}<0.05)$.

\section{Protein}

There was no difference in the protein concentrations between the experimental groups $(\mathrm{p}>0.05)$ (Table 1$)$.

\section{Correlation analysis}

A Pearson's correlation analysis was used to determine the relationship between the changes in muscle mass and volume. Changes in masseter muscle mass and volume were highly correlated $(\mathrm{R}=0.971)$ $(\mathrm{p}<0.01)$. Left signal intensity of the MRI was negatively correlated with left masseter muscle mass $(\mathrm{R}=-0.493, \mathrm{p}<0.01)$ and left masseter volume $(\mathrm{R}=-0.430, \mathrm{p}<0.05)$.

\section{Discussion}

\section{Effect of low dose formoterol}

An important finding of this study was that repeated i.m 

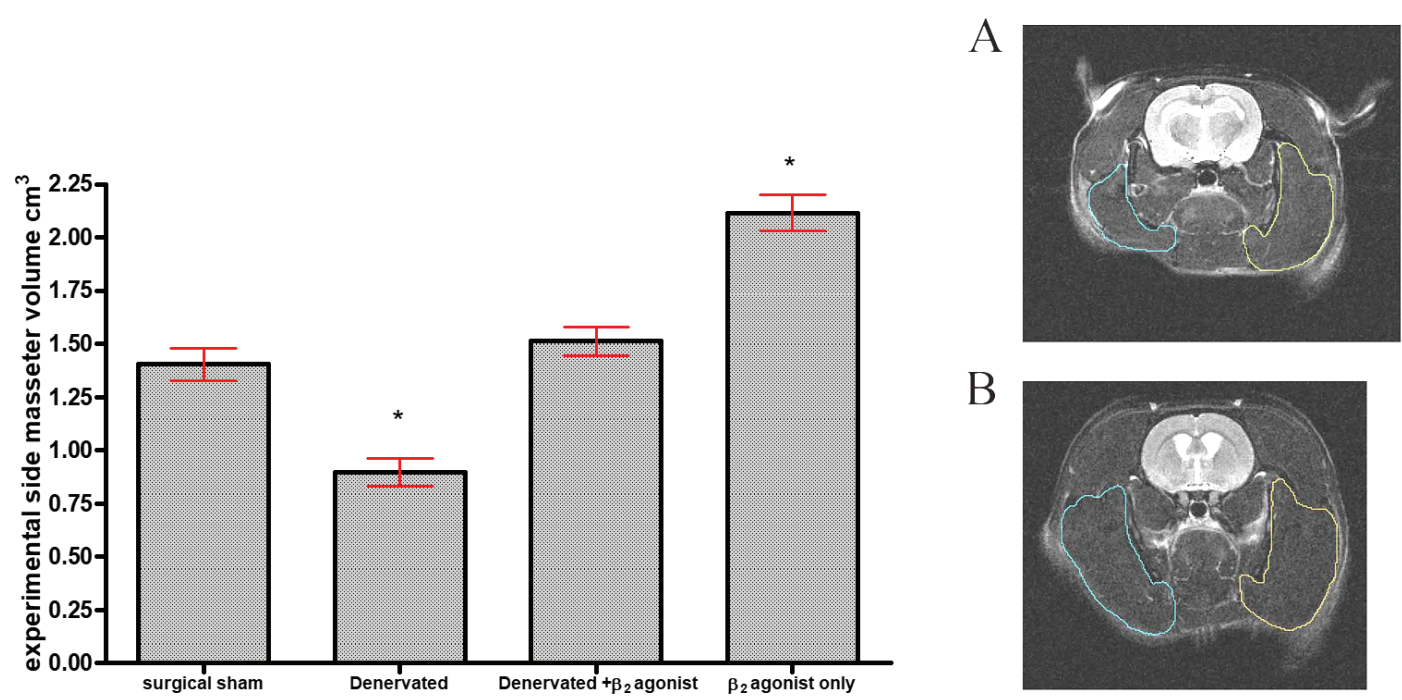

Figure 3: Graph of experimental (LHS) masseter muscle volume $\left(\mathrm{cm}^{3}\right)$. Volume significantly decreased in the denervated group, significantly increased in the $\beta 2$ agonist group, and not changed in the denervated $+\beta_{2}$ agonist group. A: an example of a section of a T2-weighted axial image taken through the level of the condylar cartilage. In the denervated group the left masseter muscle is significantly smaller than the right side. B: a similar MRI example of the $\beta 2$ agonist group. Note the larger masseter muscle in this section compared with A. ${ }^{*} p<0.05$ in experimental compared to surgical sham (control).
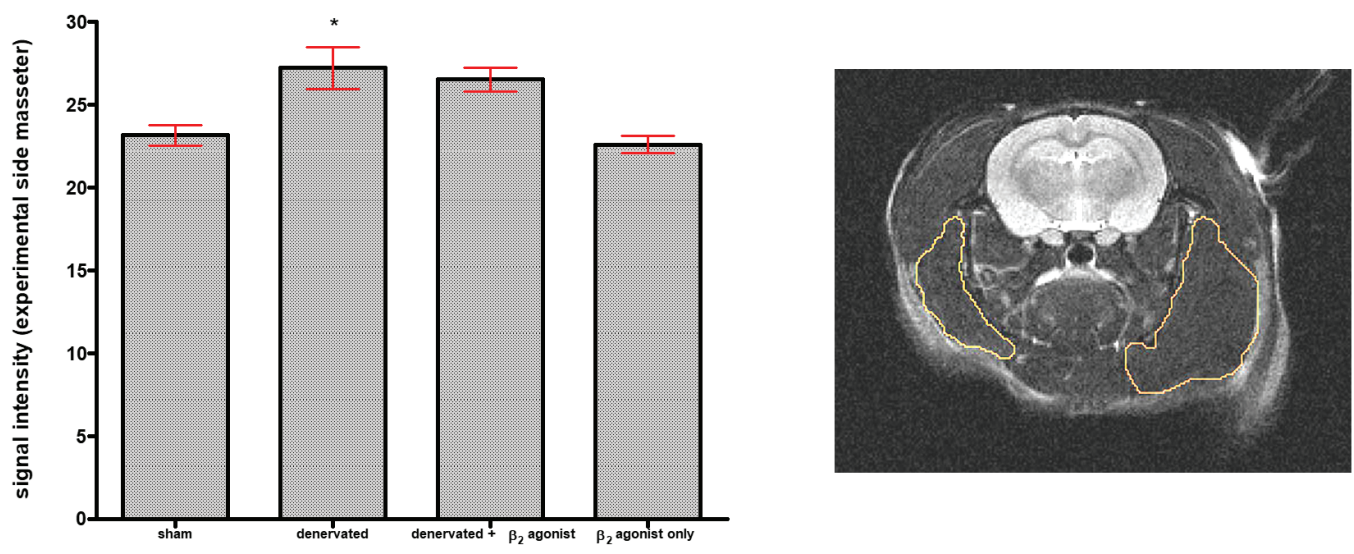

Figure 4: Graph of experimental (LHS) masseter mean signal intensity (SI).

SI significantly increased in the denervated group, and not significantly changed in the denervated $+\beta 2$ agonist group, surgical sham or $\beta$, agonist group. The picture shows an example of a denervated group rat through the section of the condylar cartilage (superomedially placed in relation to the muscle), showing the left side has a higher signal intensity (whiter) than the right side masseter muscle. ${ }^{*} p<0.05$ in experimental compared to surgical sham (control).

administration of low dose formoterol elicited significant skeletal muscle hypertrophy with no detected effects on heart or body mass. This is in contrast with the results of previous studies in which larger doses were required to cause significant skeletal and cardiac muscle hypertrophy $[14,34]$ and advances more recent findings that microgram doses of formoterol have less effect on the heart [36]. This suggests that i.m. low-dose, formoterol administration can effectively maintain or increase muscle fibre size without causing noticeable side effects.

\section{Muscular effect of denervation}

Although denervation removes about $60-70 \%$ of the muscle spindle afferents from the masticatory muscles on the experimental side, proprioceptive information from the skin, joint, teeth and remaining muscle spindle afferents provides sufficient feedback to sustain normal patterns of jaw movement [44]. The masseter muscles contribute only approximately $54 \%$ of the total masticatory muscle mass in the rat, and following denervation, the remaining masticatory muscles compensate for any loss of function [41]. Denervation resulted in significant muscle atrophy as shown by the decreased mass, volume, CSA and SI compared with controls. This decrease can be attributed to atrophy alone and not necrosis, since there was no difference in muscle protein between groups.

A significant increase in Signal Intensity (SI) was seen 8 weeks after masseter muscle denervation $(\mathrm{p}<0.05)$. This is in agreement with other studies where increased signal intensity on a MR T2-weighted 

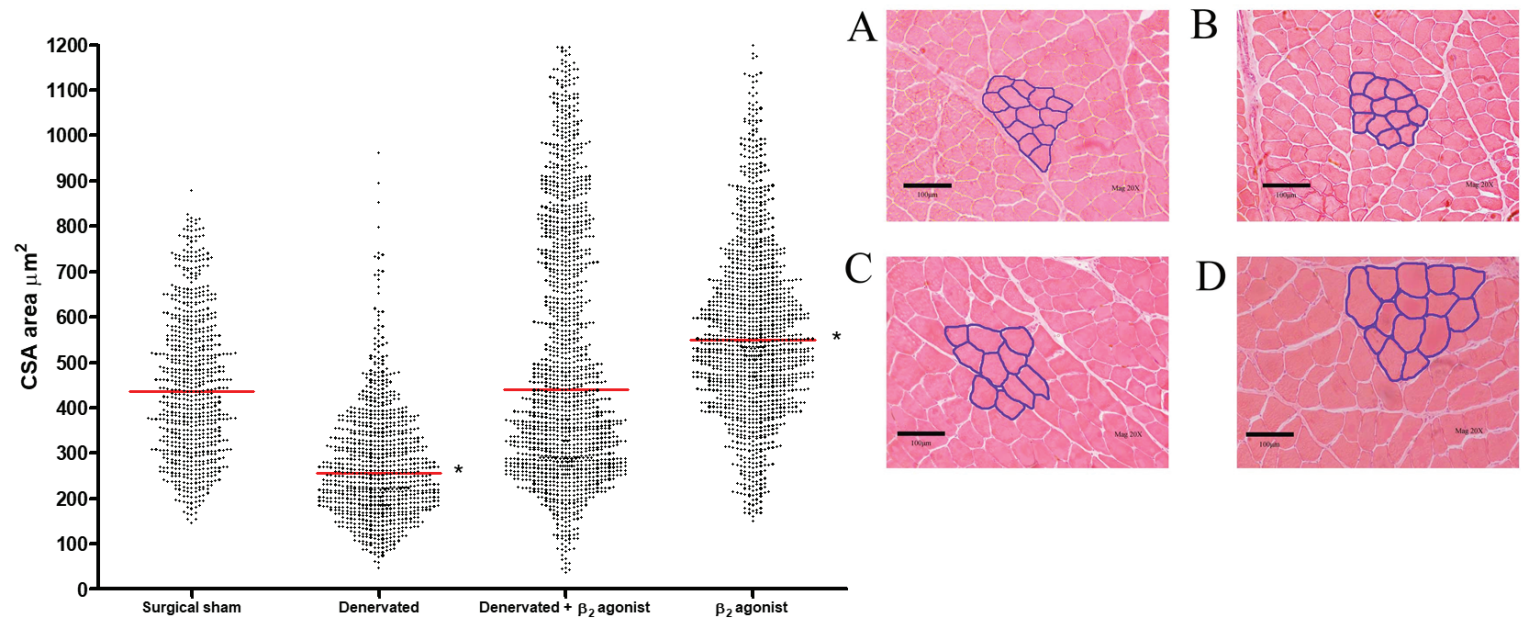

Figure 5: Graph of experimental (LHS) Cross sectional area (CSA).

CSA significantly decreased in the denervated group, significantly increased in the $\beta 2$ agonist group, and not changed in the denervated $+\beta 2$ agonist group. A: Surgical sham CSA, B: denervated group CSA, C: denervated $+\beta 2$ agonist group CSA, D: $\beta 2$ agonist only group. The red line represents the median value.

${ }^{*} p<0.05$ in experimental compared to surgical sham (control).

image was observed following denervation. SI increases following denervation can be detected as early as 1 week on T2-weighted images $[12,45-47]$. Signal intensity was highly correlated with muscle mass $(\mathrm{p}<0.01)$ and volume $(\mathrm{p}<0.05)$. Signal intensity increases on MRI may be used clinically to diagnose muscle injury, muscular dystrophies and denervation earlier than other modalities such as EMG [39]. SI levels were maintained at control values in the denervated $+\beta 2$ agonist group, as were values of mass, volume and CSA. These changes observed on MRI are further evidence that formoterol can attenuate muscle atrophy.

\section{Intramuscular $\beta 2$-agonist administration}

The muscle growth promoting effects of $\beta 2$-agonists have been studied extensively. In a previous study where clenbuterol was administered to rats via their drinking water for three weeks, masseter muscle mass was $47 \%$ greater than in untreated rats [46]. Muscle fibre diameter was increased by $27 \%$ compared with controls. Body mass also increased significantly with systemic administration. In the present study, intramuscular formoterol administration increased masseter muscle mass by $37 \%$ and CSA by $29 \%$, indicating that local formoterol administration is as effective as systemic administration in eliciting changes in masseter muscle mass and muscle fibre crosssectional area.

Interestingly, despite the $\beta 2$-agonist group masseter muscle being significantly larger in volume compared to the other experimental groups, the contralateral masseter muscle was not different $(\mathrm{p}>0.05)$. This may be, at least in part, explained by compensatory hypertrophy of the contralateral muscle in response to increased function of the left side in an attempt to maintain normal patterns of mandibular function. Mass and volume of the left masseter muscle was maintained in the denervation $+\beta 2$-agonist group; however, the contralateral side still showed a significant increase in mass and volume. This may be due to a direct "leakage" effect of the intramuscular formoterol. Although no systemic effect was found in the cardiac muscle, the i.m delivery may also have an unavoidable local effect due to the rich vascular supply of the craniofacial region. Improving the localisation of the effect of the $\beta 2$-agonist to a single muscle without iatrogenic effects on surrounding muscles could be a direction of future research.

\section{$\boldsymbol{\beta} 2$-agonists' effect on muscle wasting}

The major finding of this study was that the low-dose formoterol injected directly into skeletal muscle attenuated muscle fibre atrophy. Muscle mass, volume, CSA, SI and muscle protein was maintained at control levels following denervation and $\beta 2$-agonist administration. These data support the hypothesis that intramuscular $\beta 2$-agonist administration can attenuate denervation atrophy. The changes in muscle mass, volume and muscle fibre CSA in formoterol-treated muscles were accompanied by no changes in protein concentration. This infers that the attenuation of muscle atrophy associated with formoterol administration can be attributed to muscle hypertrophy rather than an increase in adipose tissue or inflammatory cells. Cockman MD, et al. [25] using similar MRI techniques reported that the $\beta 2$-agonist, clenbuterol, has a protective anti-atrophic effect on denervated muscle.

The exact mechanism of action for the attenuation of atrophy of formoterol has yet to be determined. $\beta 2$-agonist administration is thought to increase protein synthesis [12] and or reduce protein degradation [47]. Beitzel F, et al. [9] reported that $\beta 2$-agonist administration could enhance muscle repair and hasten functional recovery after injury [10]. Insulin-like growth factors have been reported to play a role in clenbuterol-induced hypertrophy [48], and regeneration of skeletal muscle. Other peptide growth factors, such as transforming growth factor- $\beta$ (TGF- $\beta$ ) [46], Platelet Derived Growth Factors (PDGFs), and Fibroblast Growth Factors (FGFs) may also play a role in this regenerative process by regulation of the removal of cellular debris, and activation of quiescent muscle stem cells (satellite cells) that proliferate and differentiate to form new myotubes and replace damaged cells [49]. The $\beta$-adrenergic signaling pathway represents a specific target for the treatment of muscle wasting and weakness due to its ability to promote muscle growth 
and alter fibre type proportions. It may be able to mimic innervation in denervated skeletal muscle through stimulation of neurotrophic factors and expression of the proteins myogenin and myoD, which are increased in muscles undergoing denervation induced atrophy [50]. The $\beta$-adrenergic signaling pathway is highly susceptible to chronic stimulation; however, the anti-atrophic effects of single administration of $\beta 2$-agonists are short-lived, and do not persist beyond 7 days [51], returning to control levels after 14 days [52]. In the present study, repeated i.m injections at 3 day intervals was able to ameliorate denervation atrophy over an 8 week period; however, 7 day intervals may however prove adequate.

\section{Use of MRI for studying muscle wasting}

Masseter muscle volume was increased by $50 \%$, after $\beta 2$-agonist administration and muscle volume was decreased by $56 \%$ after denervation. These changes closely match the proportional changes observed with increases in muscle mass and CSA after $\beta 2$-agonist administration, and decreasing mass and CSA after denervation. A high correlation $(\mathrm{p}<0.01)$ of experimental side muscle mass and volume $(\mathrm{R}=0.971)$ indicates that MRI can accurately measure changes in muscle volume. This could be applied to the long term observation and assessment of muscle changes in various disease states and muscle wasting in vivo. Such an approach might avoid the need to use so many rats at different experimental time points.

\section{Conclusions}

The findings of this study clearly indicate that intramuscular administration of formoterol can attenuate denervation-induced muscle atrophy. Furthermore, repeated intramuscular injection of formoterol at low doses can attenuate denervation atrophy, without effects on cardiac mass. The application of intramuscular administration of formoterol may have several possible applications in the orofacial region: preventing orofacial muscle wasting due to ageing and edentulism; preventing atrophy associated with trauma and orthognathic surgical procedures; treatment of neuromuscular conditions such as the muscular dystrophies that affect the orofacial muscles; and aiding in the control of muscle behavior during orthodontic treatment and then in the years following treatment.

\section{Conflict of Interest}

No known conflict of interest.

\section{Funding}

Australian Society of Orthodontists' Foundation for Research and Education and Melbourne Centre for Facial Disorders.

\section{Ethics}

All experiments were approved by the Animal Experimentation Ethics Committee of The University of Melbourne (AECC number 0704146.1), and Florey Institute of Neuroscience Animal Experimentation Ethics Committee (AEC number 07-067), and performed in accordance with the guidelines determined by the National Health and Medical Research Council.

\section{Acknowledgements}

The authors would like to thank the staff at the Florey Institute of Neuroscience for their assistance with Animal MRI and other technical aspects of this work.

\section{References}

1. Pepicelli A, Woods M, Briggs C (2005) The Mandibular Muscles and Their Importance in Orthodontics: A Contemporary Review. Am J Orthod Dentofacial Orthop 128: 774-780.
2. Woods MG (2017) The Mandibular Muscles in Contemporary Orthodontic Practice: A Review. Aust Dent J 62: 78-85.

3. Kane AA, Lo LJ, Christensen GE, Vannier MW, Marsh JL (1997) Relationship between bone and muscles of mastication in hemifacial microsomia. Plast Reconstr Surg 99: 990-997.

4. Ng HP, Ong SH, Hu Q, Foong KWC, Goh PS, et al. (2006) Muscles of mastication model-based MR image segmentation. Int J Comput Assist Radiol Surg 1: 137-148.

5. Raustia AM, Salonen MA, Pythinen J (1996) Evaluation of Masticatory Muscles of Edentulous Patients by Computed Tomography and Electromyography. J Oral Rehabil 23: 11-16.

6. Chan HJ, Woods M, Stella D (2008) Mandibular Muscle Morphology in Children with Different Vertical Facial Patterns: A 3-dimensional Computed Tomography Study. Am J Orthod Dentofac Orthop 133: 10e1-10e13.

7. Wong A, Woods MG, Stella D (2016) Three-dimensional Computed Tomographic Assessment of Mandibular Muscles in Growing Subjects With Different Vertical Facial Patterns. Aust Orthod J 32: 2-17.

8. Mastroianni D, Woods MG (2019) 3D-CT assessment of mandibular widths in young subjects with different underlying vertical patterns. J World Fed Orthods 8: 78-86.

9. Beitzel F, Gregorevic P, Ryall JG, Plant DR, Sillence MN, et al. (2003) Beta2-adrenoceptor Agonist Fenoterol Enhances Functional Repair of Regenerating Rat Skeletal Muscle After Injury. J Appl Physiol 96: 1385-1392.

10. Ball DI, Brittain RT, Coleman RA, Denyer LH, Jack D, et al. (1991) Salmeterol, a Novel, Long-Acting Beta 2-adrenoceptor Agonist: Characterization of Pharmacological Activity in Vitro and in Vivo. Br J Pharmacol 104: 665-671.

11. Lynch GS, Ryall JG (2008) Role of Beta-Adrenoceptor Signaling in Skeletal Muscle: Implications for Muscle Wasting and Disease. Physiol Rev 88: 729-767.

12. Choo JJ, Horan MA, Little RA, Rothwell NJ (1992) Anabolic Effects of Clenbuterol on Skeletal Muscle are Mediated by Beta 2-adrenoceptor Activation. Am J Physiol 263: E50-E56.

13. Ryall JG, Plant DR, Gregorevic P, Sillence MN, Lynch GS (2004) Beta 2-agonist Administration Reverses Muscle Wasting and Improves Muscle Function in Aged Rats. J Physiol 555: 175-188.

14. Hurley BF (1995) Age, gender, and Muscular Strength. J Gerontol A Biol Sci Med Sci 50: 41-44.

15. Argiles JM (2005) Cancer-associated Malnutrition. Eur J Oncol Nurs 9: S39-S50.

16. Giger JM, Haddad F, Qin AX, Zeng M, Baldwin KM (2005) Effect of unloading on type I myosin heavy chain gene regulation in rat soleus muscle. J Appl Physiol 98: 1185-1194.

17. Nannini LJ, Cates CJ, Lasserson TJ, Poole P (2007) Combined corticosteroid and Long-acting Beta-Agonist in One Inhaler versus Long-Acting Beta Agonists for Chronic Obstructive Pulmonary Disease. Cochrane Database Syst Rev 4: CD006829.

18. Finol HJ, Lewis DM, Owens R (1981) The Effects of Denervation on Contractile Properties or Rat Skeletal Muscle. J Physiol 319: 81-92.

19. Skura CL, Fowler EG, Wetzel GT, Graves M, Spencer MJ (2008) Albuterol Increases Lean Body Mass in Ambulatory Boys with Duchenne or Becker Muscular Dystrophy. Neurology 70: 137-143. 
20. Lynch GS, Schertzer JD, Ryall JG (2007) Therapeutic Approaches for Muscle Wasting Disorders. Pharmacol Ther 113: 461-487.

21. Fitzpatrick B, Woods M, Lynch G, Manton D (2014) The Effects of Denervation and Formoterol Administration on Facial Growth. Aust Orthod J 30: 161-168.

22. Mayne RJ, van der Poel C, Woods MG, Lynch GS (2015) Skeletal Effects of the Alteration of Masseter Muscle Function. Aust Orthod J 31: 184-194.

23. Zeman RJ, Ludemann R, Etlinger JD (1987) Clenbuterol, a Beta 2-agonist, Retards Atrophy in Denervated Muscles. Am J Physiol 252: E152-E155.

24. Maltin CA, Reeds PJ, Delday MI, Hay SM, Smite FG, et al. (1986) Inhibition and Reversal of Denervation-Induced Atrophy by the Beta-Agonist Growth Promoter, Clenbuterol. Biosci Rep 6: 811-818.

25. Cockman MD, Jones MB, Prenger MC, Sheldon RJ (2001) Magnetic Resonance Imaging of Denervation-Induced Muscle Atrophy: Effects of Clenbuterol in the Rat. Muscle Nerve 24: 1647-1658.

26. Kissel JT, McDermott MP, Mendell JR, King WM, Pandya S, et al. (2001) Randomized, Double-Blind, Placebo-Controlled Trial of Albuterol in Facioscapulohumeral Dystrophy. Neurology 57: 14341440.

27. Martineau L, Horan MA, Rothwell NJ, Little RA (1992) Salbutamol, a Beta2-adrenoceptor Agonist, Increases Skeletal Muscle Strength in Young Men. Clin Sci (Lond) 83: 615-621.

28. Libretto SE (1994) A review of the toxicology of salbutamol (Albuterol). Arch Toxicol 68: 213-216.

29. Gregorevic P, Ryall JG, Plant DR, Sillence MN, Lynch GS (2005) Chronic Beta-Agonist Administration Affects Cardiac Function of Adult but Not Old Rats, Independent of Beta-Adrenoceptor Density Am J Physiol Heart Circ Physiol 289: H344-H349.

30. Duncan ND, Williams DA, Lynch GS (2000) Deleterious Effects of Chronic Clenbuterol Treatment on Endurance and Sprint Exercise Performance in Rats. Clin Sci (Lond) 98: 339-347.

31. Carbo N, Lopez-Soriano J, Tarrago T, Gonzales O, Llovera M, et al. (1997) Comparative Effects of beta 2-adrenergic Agonists on Muscle Waste Associated With Tumour Growth. Cancer Lett 115: 113-118.

32. Ryall JG, Gregorevic P, Plant DR, Sillence MN, Lynch GS (2002) Beta 2-agonist fenoterol has greater effects on contractile function of rat skeletal muscles than clenbuterol. Am J Physiol Regul Interg Comp Physiol 283: R1386-R1394.

33. Carter WJ, Lynch ME (1994) Comparison of the Effects of Salbutamol and Clenbuterol on Skeletal Muscle Mass and Carcass Composition in Senescent Rats. Metabolism 43: 1119-1125.

34. Guhan A, Cooper S, Oborne J, Lewis S, Bennett J, et al. (2000) Systemic effects of formoterol and salmeterol: a dose-response comparison in healthy subjects. Thorax 55: 650-656.

35. Waldeck B (1996) Some Pharmacodynamic Aspects on Long-Acting Beta-Adrenoceptor Agonists. Gen Pharmacol 27: 575-580.

36. Ryall JG, Sillence MN, Lynch GS (2006) Systemic Administration of beta2-adrenoceptor Agonists, Formoterol and Salmeterol, Elicit Skeletal Muscle Hypertrophy in Rats at Micromolar Doses. Br J Pharmacol 147: 587-595.
37. Raadsheer MC, Van Eijden TM, Van Spronsen PH, Van Ginkel FC Kiliaridis S, et al. (1994) A Comparison of Human Masseter Muscle Thickness Measured by Ultrasonography and Magnetic Resonance Imaging. Arch Oral Biol 39: 1079-1084.

38. Polak JF, Jolesz FA, Adams DF (1988) Magnetic Resonance Imaging of Skeletal Muscle. Prolongation of T1 and T2 Subsequent to Denervation. Invest Radiol 23: 365-369.

39. Kikuchi Y, Nakamura T, Takayama S, Horiuchi Y, Toyama Y (2003) MR Imaging in the Diagnosis of Denervated and Reinnervated Skeleta Muscles: Experimental Study in Rats. Radiology 229: 861-867.

40. Kamath S, Venkatanarasimha N, Walsh MA, Hughes PM (2008) MRI Appearance of Muscle Denervation. Skeletal Radiol 37: 397-404.

41. Rayne J, Crawford GN (1972) The growth of the Muscles of Mastication in the Rat. J Anat 113: 391-408.

42. Dahlberg G (1940) Statistical methods for medical and biological students. Interscience Publications, New York.

43. Kikuchi M, Lu CH, Sebata M, Yamamoto Y (1978) The Mandibular Development of the Rat After the Denervation of the Masseteric Nerve. Bull Tokyo Dental Coll 19: 75-86.

44. Karlsen K (1965) The Location of Motor End Plates and the Distribution and Histological Structure of Muscle Spindles in Jaw Muscles of the Rat. Acta Odontol Scand 23: 521-547.

45. Bendszus M, Koltzenburg M, Wessig C, Solymosi L (2002) Sequential MR Imaging of Denervated Muscle: Experimental Study. Am J Neuroradiol 23: 1427-1431.

46. Akutsu S, Shimada A, Yamane A (2006) Transforming Growth Factor Betas are Upregulated in the Rat Masseter Muscle Hypertrophied by Clenbuterol, a beta2 Adrenergic Agonist. Br J Pharmacol 147: 412421.

47. Reeds PJ, Hay SM, Dorward PM, Palmer RM (1986) Stimulation of Muscle Growth by Clenbuterol: Lack of Effect on Muscle Protein Biosynthesis. Br J Nutr 56: 249-258.

48. Sneddon AA, Delday MI, Steven J, Maltin CA (2001) Elevated IGFII mRNA and Phosphorylation of 4E-BP1 and p70 (S6k) in Muscle Showing Clenbuterol-Induced Anabolism. Am J Physiol Endocrinol Metab 281: E676-E682.

49. Husmann I, Soulet L, Gautron J, Martelly I, Barritault D (1996) Growth Factors in Skeletal Muscle Regeneration. Cytokine Growth Factor Rev 7: 249-258.

50. Maltin CA, Delday MI, Campbell SP, Hesketh JE (1993) Clenbuterol Mimics Effects of Innervation on Myogenic Regulatory Factor Expression. Am J physiol 265: E176-E178.

51. Ryall JG, Schertzer JD, Alabakis TM, Gehrig SM, Plant DR, et al. (2008) Intramuscular beta2-agonist Administration Enhances Early Regeneration and Functional Repair in Rat Skeletal Muscle After Myotoxic Injury. J Appl Physiol 105: 165-172.

52. Ong A, Woods MG, Lynch GS (2020) Alteration of muscle size and strength with the administration of a Beta agonist. Int J Dent Oral Health 6. 